\title{
Escalation Strategies in the Treatment of Refractory Myasthenia Gravis
}

\section{(ㄷ) (1) (ㅇ) $\ominus$}

\author{
Authors \\ Sarah Hoffmann ${ }^{1,2}$, Andreas Meisel1, 2

\section{Affiliations \\ 1 Klinik für Neurologie, Charité - Universitätsmedizin Berlin \\ 2 NeuroCure Clinical Research Centre (NCRC), Charité - Universitätsmedizin Berlin}

Key words

myasthenia gravis, refractory MG, immunosuppression, rituximab, eculizumab

\author{
Bibliography \\ DOI https://doi.org/10.1055/s-0043-125342 \\ Neurology International Open 2018; 2: E56-E59 \\ (c) Georg Thieme Verlag KG Stuttgart · New York \\ ISSN 2511-1795
}

\author{
Correspondence \\ Dr. med. Sarah Hoffmann \\ Klinik für Neurologie \\ Charité - Universitätsmedizin Berlin \\ Charitéplatz 1 \\ 10117 Berlin \\ Germany \\ sarah.hoffmann@charite.de
}

\section{Introduction}

Most myasthenia gravis (MG) patients are sufficiently treated with expanded standard therapy; 10 to $15 \%$ of cases are therapy-refractory. Such situations are very stressful for patients and represent a major challenge in the treatment of myasthenia gravis. There is to date no generally-accepted definition of therapy-refractory myasthenia gravis. It is broadly accepted that these are generalized courses of the disease which despite (expanded) standard therapy consisting of acetylcholinesterase inhibitors, steroids and usually at least two steroid-sparing long-term immunosuppressants in sufficient dose and duration exhibit moderate to severe functional impairment (see German article by Urban et al., Therapiestandards und individualisierte Therapie bei der Myasthenia gravis (English: Therapeutic Standards and individualized Therapy for Myasthenia Gravis)) [1]. Current international consensus guidelines recommend treating treatment-refractory MG cases in specialized centers with sufficient expertise, as well as therapy escalation with regular IVIg administration, plasmapheresis or immunoadsorption at intervals [1]. These procedures are approved for the treatment of myasthenic crisis in Germany; IVIg is also approved for off-label use in cases of severe exacerbation (refer to German article by Janzen R., Janzen R., Off-Label-Use bei Seltenen Erkrankungen: Myasthenia gravis, Lambert-Eaton myasthenisches Syndrom und kongeni- tale myasthenische Syndrome (English: Off-label Use for rare Diseases: Myasthenia Gravis, Lambert-Eaton Myasthenic Syndrome and congenital myasthenic Syndromes)), but not for interval therapy (refer to German article by Schroeder et. al. 2017, Myasthenie - Exazerbation und Krise (English: Myasthenia - Exacerbation and (risis)). Repeated use of these procedures as escalation therapy is likewise considered a feature of refractory courses of the disease. Serious side effects and clinically relevant contraindications of standard therapeutics must also be taken into account (see information box). Therapy-refractory MG patients are more likely to be female, younger (25 to 50 years), thymoma-associated, or anti-MuSK-positive.

This article provides an overview of reserve drugs used as well as ongoing intervention studies for the treatment of refractory MG. On the one hand, the evaluation of these drugs must take into account that the majority of MG patients can be treated satisfactorily with (expanded) standard therapy, despite the fact that the available data is insufficient even for the approved drugs prednisone, prednisolone, azathioprine and mycophenolate mofetil (the latter used off-label in the case of ineffectiveness or intolerance of azathioprine therapy) (refer to article by Urban et al., Op. cit.). On the other hand, the reserve drugs discussed below have been primarily used in therapy-refractory MG patients, so that it can only be as- 
sumed that their effectiveness in non-treatment-resistant MG patients is at least as good as that of refractory patients.

\section{Rituximab}

The monoclonal anti-CD20 antibody rituximab has been in off-label use in the treatment of MG for several years. The rationale for this therapy is the elimination of B lymphocytes, the progenitor cells of antibody-producing plasma cells. To date, there are a number of case series and uncontrolled studies indicating positive effects in MG patients who are refractory to standard medications $[2,3]$. Although positive effects have primarily been described in anti-MuSK-positive patients, it is becoming increasingly clear that this is also true for anti-AChR-positive as well as seronegative MG patients (with absent evidence of antibodies) [4]. Two systematic reviews on the use of rituximab in refractory MG cases report a response rate of $83.9 \%$ and $44 \%$, respectively $[2,4]$. Although lorio et al. found comparable response rates independent of antibody status [4], Tandan et al. reported significantly higher response rates among MuSK-positive compared to AchR Ab-positive patients ( $72 \%$ vs. $30 \%$ ) [2]. Currently, one Phase II and one Phase III trial on the effect of rituximabin MG are ongoing (ClinicalTrials.gov Identifier: NCT02110706, NCT02950155). At this time, two different therapy regimes are being used:

1. Administration of $2 \times 1000 \mathrm{mg}$ absolute dose at a 14-day interval or

2. Weekly administration of $375 \mathrm{mg} / \mathrm{m} 2$ body surface for 4 successive weeks

Each regimes is continued by follow-up doses at an interval of approx. 6 months. It is unclear how often the therapy should be repeated. Current data indicate that this therapy should be performed at least three times [5]. In addition, immune markers (e.g., regulatory $B$ cells, memory $B$ cells) are increasingly being investigated for guiding therapy [6]. On the whole, Rituximab has a favorable side effect profile [4]. However, 2 cases of progressive multifocal leukoencephalopathy in MG patients during rituximab therapy have been described [7,8].

\section{Eculizumab}

Eculizumab is a monoclonal antibody directed against complement factor $C 5$. Eculizumab is approved for the treatment of paroxysmal nocturnal hemoglobinuria and has also been used successfully in atypical hemolytic uremic syndrome [9]. The complement system plays an important role in the AChR antibody-induced destruction mechanism of the neuromuscular junction. This involvement of the complement system depends on the IgG subtype; the AChR antibody belongs to the $\mathrm{lgG} 1$ subtype, while the anti-MuSK is an $\operatorname{lgG} 4$ antibody subtype in which the complement system plays no role. Based on these pathophysiological considerations, eculizumab was tested in 14 treatment-refractory anti-AChR-positive MG patients in an investigator-initiated, randomized, double-blind, placebo-controlled, cross-over Phase II study. Compared to placebo, there was a significant improvement in the quantitative myasthenia gravis score (QMG) of patients treated with eculizumab [10]. The subsequent manufacturer-sponsored, randomized, double-blind, placebo-controlled Phase III (REGAIN) study on the use of eculizumab in refractory anti-AchR-positive MG patients enrolled 126 patients (ClinicalTrials.gov Identifier: NCT01997229). As primary endpoint, the MG-ADL score (myasthenia-specific score for daily living activity) was examined using the worst-rank method, which was missed with a p-value of 0.0698 . The secondary endpoints (such as QMG, MG-QOL) were significantly improved in the worst-rank procedure, as well as responder analyses and sensitivity analyses [11]. There was no significant difference between the two groups with respect to side effects. Based on the available data, the European Commission approved the use of eculizumab in refractory generalized, anti-AChR-positive myasthenia gravis. Eculizumab is administrated parenterally, in the induction phase (weeks 1-4) at a dose of $900 \mathrm{mg}$ weekly, in the maintenance phase (from week 5) at a dose of $1200 \mathrm{mg}$ every 2 weeks. A decision to continue therapy should be made based on an evaluation after 6 and no later than 12 weeks of treatment of whether a significant improvement in the Quantitative Myasthenia Gravis (QMG) Score as well as daily activity (Myasthenia Gravis Activities of Daily Living, MG-ADL) was achieved. Similar to other immunosuppressants used for MG, it is currently unclear how long eculizumab should be administered. In the course of the REGAIN study, eculizumab was given for 26 weeks. The study participants had the opportunity to continue treatment as part of a currently ongoing open label extension study (ClinicalTrials.gov Identifier: NCT02301624). In the prior Phase II cross-over study, patients initially treated with eculizumab experienced worsening of the symptoms during the placebo study phase without reaching baseline QMG scores. This might indicate a longer duration of eculizumab effectiveness and may justify an attempt to discontinue therapy, which should be performed after about 12 months; therapy should be continued if the symptoms worsen significantly. By consensus, the medical advisory board of the German Myasthenia gravis Society recently presented concrete recommendations for the definition of therapy-refractory generalized myasthenia gravis as well as the indication and proceedings for therapy with eculizumab [12]. Since life-threatening and fatal meningococcal infections have occurred in connection with eculizumab therapy, patients must be vaccinated against meningococci prior to treatment (as was done in the studies mentioned above). For medical reasons, the indication for therapy must be assessed very carefully. This includes the weighing of the individual therapy of severely affected MG patients against the very high treatment costs of eculizumab.

INFORMATION BOX: CHARACTERISTICS OF THERAPY-REFRACTORY MYASTHENIA GRAVIS

- chronic courses with moderate to severe symptoms and/ or functional impairment

AND

- ineffective (expanded) standard therapy

- repeated myasthenic crises or severe exacerbation

- repeated need for therapy escalation with IVIg, plasmapheresis or immunadsorption OR

- standard therapy with unacceptable side effects

- contraindication for standard therapies due to comorbidities 
- Table 1 Current clinical studies of immunomodulator therapy of myasthenia gravis.

\begin{tabular}{|c|c|c|}
\hline Preparation & Action mechanism & $\begin{array}{l}\text { Study phase } \\
\text { ClinicalTrials.gov identifier: }\end{array}$ \\
\hline Bortezomib & Proteasome inhibitor with plasma cell depletion & $\begin{array}{l}\text { Phase II } \\
\text { NCT02102594 }\end{array}$ \\
\hline Belimumab & Monoclonal antibody against B cell activating factor (BAFF) & $\begin{array}{l}\text { Phase II } \\
\text { NCT01480596 }\end{array}$ \\
\hline CFZ533 & $\begin{array}{l}\text { Monoclonal antibody against CD } 40 \text {, mainly inhibiting the antigen-specific } \\
\text { activation of naive B lymphocytes }\end{array}$ & $\begin{array}{l}\text { Phase II } \\
\text { NCT02565576 }\end{array}$ \\
\hline ARGX-113 & $\begin{array}{l}\text { Monoclonal antibody against neonatal IgG Fc receptor, leading to faster } \\
\text { degradation of } \operatorname{lgG}\end{array}$ & $\begin{array}{l}\text { Phase II } \\
\text { NCT02965573 }\end{array}$ \\
\hline Rozanolixizumab (UCB7665) & $\begin{array}{l}\text { Monoclonal antibody targeting Fc fragment of IgG receptor and transporter } \\
\text { (FCGRT) }\end{array}$ & $\begin{array}{l}\text { Phase II } \\
\text { NCT03052751 }\end{array}$ \\
\hline CV-MG01 & Vaccination to form anti-idiotypic antibodies against AchR Ab and T cell receptors & $\begin{array}{l}\text { Phase lb } \\
\text { NCT02609022 }\end{array}$ \\
\hline Abatacept & $\begin{array}{l}\text { Inhibition of T cell activation by binding to the CD80 and CD86 molecules of } \\
\text { antigen-presenting cells }\end{array}$ & $\begin{array}{l}\text { Phase I } \\
\text { NCT03059888 }\end{array}$ \\
\hline
\end{tabular}

\section{High-dose Cyclophosphamide and Stem Cell Therapy}

High-dose cyclophosphamide therapy has been used since the 1960 s in the treatment of MG. Several case series as well as a randomized, double-blind study with 23 patients show positive effects in the treatment of refractory cases [13-16]. Current international consensus guidelines likewise recommend the use of cyclophosphamide for the treatment of refractory MG cases [1]. However, data from long-term observations also show a high rate of complications of immune ablative therapy [17]. Therefore, this therapy is reserved for the most severely affected refractory MG cases and only after the above-mentioned therapies have been exhausted.

Autologous stem cell transplantation (SCT) should likewise be used as a last resort due to the relatively high mortality rate of $6-8 \%$. Autologous SCT in refractory MG patients has been repeatedly performed successfully; a potential cure of MG is discussed in a recent case series of 7 patients with complete remission after autologous SCT [18]. However, this was a retrospective case collection and none of the patients had previously been treated with rituximab or eculizumab. In this regard, it is interesting to note that AChR antibodies have been detected in up to $20 \%$ of allogenic stem cell transplanted patients due to other indications [19], although only few of them develop myasthenic symptoms [20].

\section{SUMMARY}

Both nationally and internationally, rituximab has increasingly been used in the treatment of refractory myasthenia gravis. The experience in myasthenia centers corresponds to the results in the literature, i. e., refractory MG patients with AChR antibodies, MuSK antibodies as well as seronegative patients improve under this therapy. However, sufficiently supporting study evidence is missing so far. Such evidence is now available for eculizumab, which has led to its approval for patients with anti-AChR-positive generalized refractory myasthenia gravis. Neither high-dose cyclophosphamide nor stem cell therapy play a role in the German medical care system; due to the very unfavorable side effect profile these therapies should be reserved for the extremely rare MG cases that do no respond to the above-mentioned therapies.

\section{Outlook}

There are currently several immunosuppressive or immunomodulatory drugs in different clinical study phases, the mechanisms of action are summarized in $>$ Table $\mathbf{1}$. Apart from the belimumab study, all studies are still in progress. Based on the available study registry information on the primary endpoint and the secondary endpoints, belimumab is not effective in the treatment of refractoryMG. 


\section{Conflict of Interest}

A. Meisel is a member of the medical advisory board of the German Myasthenia Society. He has received lecture fees from Grifols, Novartis and Alexion, for consulting services at Axion, as well as financial support for an Octapharma project. S. Hoffmann declares no conflicts of interest.

\section{References}

[1] Sanders DB, Wolfe Gl, Benatar M et al. International consensus guidance for management of myasthenia gravis: Executive summary. Neurology 2016; 87: 419-425

[2] Tandan R, Hehir MK 2nd, Waheed W et al. Rituximab treatment of myasthenia gravis: A systematic review. Muscle Nerve 2017; 56: 185-196

[3] Anderson D, Phan C, Johnston WS et al. Rituximab in refractory myasthenia gravis: A prospective, open-label study with long-term follow-up. Ann Clin Transl Neurol 2016; 3: 552-555

[4] Iorio R, Damato V, Alboini PE et al. Efficacy and safety of rituximab for myasthenia gravis: a systematic review and meta-analysis. J Neurol 2015; 262: 1115-1119

[5] Robeson KR, Kumar A, Keung B et al. Durability of the rituximab response in acetylcholine receptor autoantibody-positive myasthenia gravis. JAMA Neurol 2017; 74: 60-66

[6] Lebrun C, Bourg V, Bresch S et al. Therapeutic target of memory B cells depletion helps to tailor administration frequency of rituximab in myasthenia gravis. J Neuroimmunol 2016; 298: 79-81

[7] Afanasiev V, Demeret $S$, Bolgert $F$ et al. Resistant myasthenia gravis and rituximab: A monocentric retrospective study of 28 patients. Neuromuscul Disord 2017; 27: 251-258

[8] Kanth KM, Solorzano GE, Goldman MD. PML in a patient with myasthenia gravis treated with multiple immunosuppressing agents. Neurol Clin Pract 2016; 6: e17-e19

[9] Braune SA, Wichmann D, von Heinz MC et al. Clinical features of critically ill patients with Shiga toxin-induced hemolytic uremic syndrome. Crit Care Med 2013; 41: 1702-1710
[10] Howard JF Jr., Barohn RJ, Cutter GR et al. A randomized, double-blind, placebo-controlled phase II study of eculizumab in patients with refractory generalized myasthenia gravis. Muscle Nerve 2013; 48: $76-84$

[11] Howard JF Jr., Utsugisawa K, Benatar M et al. Safety and efficacy of eculizumab in anti-acetylcholine receptor antibody-positive refractory generalised myasthenia gravis (REGAIN): A phase 3, randomised, double-blind, placebo-controlled, multicentre study. Lancet Neurol 2017; 16: 976-986

[12] Ärztlicher Beirat der Deutschen Myasthenie Gesellschaft (DMG). Ärztlicher Beirat zu Eculizumab-Therapie [online]. Available at: http:// www.dmg-online.de/aerztlicher-beirat-zu-eculizumab-therapie/ Accessed 18.12.2017

[13] De Feo LG, Schottlender J, Martelli NA et al. Use of intravenous pulsed cyclophosphamide in severe, generalized myasthenia gravis. Muscle Nerve 2002; 26: 31-36

[14] Drachman DB, Adams RN, Hu R et al. Rebooting the immune system with high-dose cyclophosphamide for treatment of refractory myasthenia gravis. Ann N Y Acad Sci 2008; 1132: 305-314

[15] Gladstone DE, Brannagan TH 3rd, Schwartzman RJ et al. High dose cyclophosphamide for severe refractory myasthenia gravis. J Neurol Neurosurg Psychiatry 2004; 75: 789-791

[16] Buzzard KA, Meyer N], Hardy TA et al. Induction intravenous cyclophosphamide followed by maintenance oral immunosuppression in refractory myasthenia gravis. Muscle Nerve 2015; 52: 204-210

[17] Nagappa M, Netravathi M, Taly AB et al. Long-term efficacy and limitations of cyclophosphamide in myasthenia gravis. J Clin Neurosci 2014; 21: 1909-1914

[18] Bryant A, Atkins H, Pringle CE et al. Myasthenia gravis treated with autologous hematopoietic stem cell transplantation. JAMA Neurol 2016; 73: 652-658

[19] Smith Cl, Hammarstrom L, Lefvert AK. Bone-marrow grafting induces acetylcholine receptor antibody formation. Lancet 1985; 1: 978

[20] Tsutsumi Y, Kamiishi T, Kikuchi R et al. Myasthenia gravis after allogeneic bone marrow transplantation: A case report and literature review. Hematol Oncol Stem Cell Ther 2017, doi:10.1016/j. hemonc.2017.04.001 